\begin{tabular}{|c|l|}
\hline Title & Size selective photocatalytic reactions by titanium(iv) oxide coated with a hollow silica shell in aqueous solutions \\
\hline Author(s) & $\begin{array}{l}\text { Ikeda, Shigeru; Kobay ashi, Hidey uki; Ikoma, Y oshimitsu; Harada, Takashi; Torimoto, Tsukasa; Ohtani, Bunsho; } \\
\text { Matsumura, Michio }\end{array}$ \\
\hline Citation & $\begin{array}{l}\text { Physical Chemistry Chemical Physics, 9(48), 6319-6326 } \\
\text { https://doi.org/10.1039/0709891j }\end{array}$ \\
\hline Issue Date & 2007 \\
\hline Doc URL & http://hdl.handle.net/2115/48666 \\
\hline Rights & Phys. Chem. Chem. Phys., 2007, 9, 6319-6326- Reproduced by permission of the PCCP Owner Societies \\
\hline Type & article (author version) \\
\hline File Information & PCCP9_6319.pdf \\
\hline
\end{tabular}

Instructions for use 


\section{Shigeru Ikeda}

Research Center for Solar Energy Chemistry, Osaka University, 1-3 Machikaneyama, Toyonaka 560-8531, Japan

TEL: +81-6-6850-6696 FAX: +81-6-6850-6699

E-mail: sikeda@chem.es.osaka-u.ac.jp

Size-Selective Photocatalytic Reactions by Titanium(IV) Oxide Coated with a Hollow Silica Shell in Aqueous Solutions

Shigeru Ikeda, ${ }^{*}{ }^{\ddagger}$ Hideyuki Kobayashi,${ }^{\ddagger}$ Yoshimitsu Ikoma ${ }^{\ddagger}$ Takashi Harada, ${ }^{\ddagger}$ Tsukasa Torimoto, ${ }^{\dagger}$ Bunsho Ohtani ${ }^{\S}$, and Michio Matsumura ${ }^{\ddagger}$

$\$$ Research Center for Solar Energy Chemistry, Osaka University, 1-3 Machikaneyama, Toyonaka 560-8531, Japan

${ }^{\dagger}$ Department of Crystalline Materials Science, Graduate School of Engineering, Nagoya University, Furo, Chikusa, Nagoya 464-8603, Japan

$\S$ Catalysis Research Center, Hokkaido University, Sapporo 001-0021, Japan

A novel core-shell composite photocatalyst, commercially available titanium(IV) oxide $\left(\mathrm{TiO}_{2}\right)$ particles directly incorporated into a hollow silica shell, was fabricated by successive coating of $\mathrm{TiO}_{2}$ with a carbon layer and a silica layer followed by heat treatment to remove the carbon layer. The composite induced efficient photocatalytic reactions when relatively small substrates were used, such as methanol dehydration and decomposition of acetic acid, without any reduction in the intrinsic activity of original $\mathrm{TiO}_{2}$ but did not exhibit efficient photocatalytic activity for decomposition of large substrates, methylene blue and polyvinylalcohol. The unique size-selective properties of 
the composites are due to their structural characteristics, i.e., the presence of a pore system and a void space in the silica shell and between the shell and medial $\mathrm{TiO}_{2}$ particles, respectively. The loading of alkylsilyl groups on the surface of the composite led to highly photostable floatability: the floated sample also induced efficient photocatalytic reaction for decomposition of acetic acid while retaining floatation at the gas-water interface.

\section{Introduction}

Photocatalysis is a redox reaction driven by photoexcited electrons ( $\mathrm{e}^{-}$) in the conduction band and simultaneously generated positive holes $\left(\mathrm{h}^{+}\right)$in the valence band of semiconducting materials when photoirradiated with light of energy greater than the band gap between the valence and conduction bands. ${ }^{1}$ In the presence of molecular oxygen $\left(\mathrm{O}_{2}\right)$ under ordinary atmospheric conditions, the target of reduction by $\mathrm{e}^{-}$is limited to $\mathrm{O}_{2}$, and proposed resulting products, superoxide anion, ${ }^{2}$ hydroperoxy and/or hydroxyl radicals, ${ }^{3,4}$ are all oxidants. Thus, the photocatalytic reaction under aerated conditions is oxidation of substrates by both $\mathrm{h}^{+}$and the oxidants derived from $\mathrm{e}^{-}$. This is one of the main reasons for the potential environmental applications of photocatalysis, i.e., complete oxidation and mineralization of organic pollutants in air and water. ${ }^{5-8}$

Among the many semiconducting materials, titanium(IV) oxide $\left(\mathrm{TiO}_{2}\right)$ has proven to be the most suitable photocatalyst due to its strong oxidizing power, biological and chemical inertness, cost-effectiveness, and long-term stability against photocorrosion and chemical corrosion. Besides the conventional photocatalytic oxidation properties described above, band-gap excitation of $\mathrm{TiO}_{2}$ in an aerobic condition has also been shown to induce conversion of surface wetability, leading to a highly hydrophilic surface. ${ }^{9-11}$ These specific properties of $\mathrm{TiO}_{2}$ have resulted in commercial applications to a self-cleaning coating on external walls of buildings and glass materials. ${ }^{6,7}$ 
While photooxidation processes on $\mathrm{TiO}_{2}$ photocatalysis are nonselective owing to the strong oxidation ability as described above, selective oxidation of certain chemical bonds in a reaction substrate and degradation of only a given component from a mixture are other versatile and intriguing subjects because they open new potential fields of applications: the former enables construction of environmentally benign organic reaction systems and the latter would be useful for separation processes or the selective elimination of pollutant molecules from a mixture. Therefore, numerous studies have been conducted and have shown induction selective oxidative conversion of certain organic substrates by appropriate selection of the solvent and other reaction conditions. ${ }^{12-15}$ However, by using ordinal $\mathrm{TiO}_{2}$ particles, the number of substrates to achieve sufficient conversion and selectivity are limited, and thus it is of great importance to control activity of the $\mathrm{TiO}_{2}$ photocatalysts by modification of their bulk and/or surface properties in compliance with different needs.

One typical approach for the construction of selective photocatalytic systems is the use of photocatalysts with defined nanostructures such as mesoporous $\mathrm{TiO}_{2}$ as well as nanocrystalline $\mathrm{TiO}_{2}$ particles or isolated $\mathrm{TiO}_{2}$ species dispersed onto inorganic supports with periodic pore systems and high surface areas. ${ }^{16-22}$ Although various examples have been reported, this approach often includes a problem of difficulty to fabricate a $\mathrm{TiO}_{2}$ photocatalyst with sufficient activity comparable to that of commercially available $\mathrm{TiO}_{2}$. From the viewpoint of practical utility, therefore, the use of commercially available $\mathrm{TiO}_{2}$ having sufficient photocatalytic performance is more advantageous than the use of such synthesized $\mathrm{TiO}_{2}$ photocatalysts. One of the ordinal methods to provide the commercial $\mathrm{TiO}_{2}$ selective photocatalytic functions is encapsulation of $\mathrm{TiO}_{2}$ particles into porous substances. ${ }^{23-26}$ However, composites obtained by this method have a propensity to decrease intrinsic photocatalytic activity of the medial $\mathrm{TiO}_{2}$ due to the surface coverage of these substances.

Recently, we have reported fabrication of a novel core-shell composite 
photocatalyst, commercially available $\mathrm{TiO}_{2}$ particles directly incorporated into a hollow silica shell $\left(\mathrm{SiO}_{2} /\right.$ void $\left./ \mathrm{TiO}_{2}\right) .{ }^{27}$ It has been shown that the composite has size-selective properties in photodecomposition of organics, i.e., $\mathrm{SiO}_{2} /$ void $/ \mathrm{TiO}_{2}$ exhibited photocatalytic activity for decomposition of relatively small substrates but did not have activity for decomposition a large molecule. Moreover, the notable feature of this photocatalytic system is that it retains intrinsic activity of original $\mathrm{TiO}_{2}$ for small substrates due to the presence of a void space between the $\mathrm{TiO}_{2}$ core and the hollow silica shell.

In our previous paper, we briefly reported the structural features and size-selective photocatalytic functions of $\mathrm{SiO}_{2} /$ void $/ \mathrm{TiO}_{2}$. In this paper, we show the details of structural characteristics of the material and photocatalytic activity for various substrates in aqueous systems. Possible control of hydrophobicity of the silica shell, leading to stable floatability on aqueous solutions under photoirradiation, is also discussed.

\section{Experimental}

\section{Materials}

Commercial $\mathrm{TiO}_{2}$ samples (Ishihara ST-41 and Degussa P-25, supplied by Ishihara Sangyo and Nihon Aerosil, respectively) were used in this study. Other commercial chemicals were of the highest available grade and were used without further purification. Laboratory-grade water was prepared by using a Milli-Q pure water system (Yamato-Millipore).

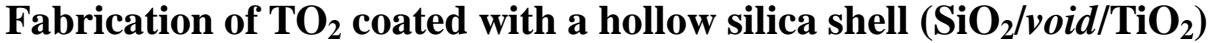

To $80 \mathrm{~cm}^{3}$ of aqueous glucose solution $\left(0.5 \mathrm{~mol} \mathrm{dm}^{-3}\right)$ was added $0.2 \mathrm{~g}$ of $\mathrm{TiO}_{2}$, and the suspension was placed in a Teflon-sealed autoclave and maintained at $453 \mathrm{~K}$ for 
$6 \mathrm{~h}$. The resulting powder was isolated by filtration, washed with water and ethanol, and heated at $873 \mathrm{~K}$ under evacuation for $2 \mathrm{~h}$. Then $0.2 \mathrm{~g}$ of carbon-coated $\mathrm{TiO}_{2}$ thus-obtained $\left(\mathrm{C} / \mathrm{TiO}_{2}\right)$ was stirred in an ethanolic solution $\left(10 \mathrm{~cm}^{3}\right)$ containing 0.42 mmol of $n$-(2-aminoethyl)-3-aminopropyltrimethoxysilane (AEAPS) for $2 \mathrm{~h}$ at room temperature. The resulting AEAPS-treated sample was put into an ethanolic solution (14 $\mathrm{cm}^{3}$ ) containing $2.2 \mathrm{mmol}$ tetraethyl orthosilicate (TEOS), $0.5 \mathrm{~cm}^{3}$ aqueous ammonia $(28 \%)$ and $2 \mathrm{~cm}^{3}$ water. After agitating the suspension for $1.5 \mathrm{~h}$ at room temperature, the solid part was collected by centrifugation, washed with water several times, and dried at $383 \mathrm{~K}$ under vacuum to yield $\mathrm{TiO}_{2}$ particles covered successively with a carbon layer and a silica layer $\left(\mathrm{SiO}_{2} / \mathrm{C} / \mathrm{TiO}_{2}\right)$. Finally, the $\mathrm{SiO}_{2} / \mathrm{C} / \mathrm{TiO}_{2}$ sample was heated at $873 \mathrm{~K}$ for $3 \mathrm{~h}$ in air to remove carbon components. In order to study the effectiveness of surface amino-functionalization during the coating with a silica layer on $\mathrm{C} / \mathrm{TiO}_{2}$, we also attempted to coat the $\mathrm{C} / \mathrm{TiO}_{2}$ sample with a silica layer without treatment with AEAPS. As a reference, $\mathrm{TiO}_{2}$ directly coated with silica $\left(\mathrm{SiO}_{2} / \mathrm{TiO}_{2}\right)$ was also prepared by following the procedure developed by Graf et al. with slight modifications. ${ }^{28}$ Briefly, $0.2 \mathrm{~g}$ of $\mathrm{TiO}_{2}$ was stirred in an aqueous poly(vinylpyrrolidone) (PVP) solution (2.3 mmol dm${ }^{-3}, 18 \mathrm{~cm}^{3}$ ) for $12 \mathrm{~h}$. The PVP-adsorbed $\mathrm{TiO}_{2}$ powder as-obtained was stirred in an ethanolic solution $\left(14 \mathrm{~cm}^{3}\right)$ containing $0.5 \mathrm{mmol}$ tetraethyl orthosilicate (TEOS), 0.5 $\mathrm{cm}^{3}$ aqueous ammonia (28\%) and $2 \mathrm{~cm}^{3}$ water for $1.5 \mathrm{~h}$. The resulting powder was collected by centrifugation and heated at $873 \mathrm{~K}$ for $3 \mathrm{~h}$ in air.

\section{Modification of particle surfaces with an alkylsilylation agent}

Functionalization of external surfaces of $\mathrm{TiO}_{2}, \mathrm{SiO}_{2} /$ void $/ \mathrm{TiO}_{2}$ and $\mathrm{SiO}_{2} / \mathrm{TiO}_{2}$ with alkylsilyl groups was carried out as follows. To $10 \mathrm{~cm}^{3}$ of toluene containing $2.5 \mathrm{mmol}$ of octadecyltrichlorosilane (ODS) was added $50 \mathrm{mg}$ of $\mathrm{TiO}_{2}, \mathrm{SiO}_{2} /$ void $/ \mathrm{TiO}_{2}$ or $\mathrm{SiO}_{2} / \mathrm{TiO}_{2}$. After the mixtures had been shaken for $1 \mathrm{~h}$ at $333 \mathrm{~K}$, the suspensions were centrifuged and washed with ethanol $\left(20 \mathrm{~cm}^{3}\right)$ to remove unreacted ODS and a 
by-product, hydrogen chloride. The precipitates were then dried at $373 \mathrm{~K}$ overnight. The resulting powders are designated as o- $\mathrm{TiO}_{2}$ and o-SiO $2 /$ void $/ \mathrm{TiO}_{2}$, respectively.

\section{Characterization and analytical procedures}

The morphology of the particles was examined using a Hitachi S-5000 FEG scanning electron microscope (SEM) at a voltage of $20 \mathrm{kV}$. Thermogravimetry-differential thermal analysis (TG-DTA) was conducted using a Bruker 2000A TG-DTA apparatus in air from room temperature to $1073 \mathrm{~K}$ with a heating ramp of $10 \mathrm{~K} \mathrm{~min}^{-1}$. The structures of the particles were also confirmed by transmission electron microscopy (TEM) using a Hitachi H-9000 transmission electron microscope. Powder X-ray diffraction (XRD) was measured using a Rigaku MiniFlex X-ray diffractometer ( $\mathrm{CuK} \alpha$, Ni filter). Nitrogen $\left(\mathrm{N}_{2}\right)$ sorption isotherms at $77 \mathrm{~K}$ were obtained on a Quantachrome AUTOSORB-1 automated gas sorption system after drying samples at $473 \mathrm{~K}$ for $2 \mathrm{~h}$. The amounts of siliceous components in the $\mathrm{SiO}_{2} /$ void $/ \mathrm{TiO}_{2}$ samples were determined by using a Nippon Jarrel-Ash ICPAP-575 Mark II inductively coupled plasma atomic emission spectrometer (ICP-AES). For the measurement, silicon components on the samples were dissolved by dispersing the samples in a $0.1 \mathrm{~mol} \mathrm{dm}^{3} \mathrm{NaOH}$ solution for 6 $\mathrm{h}$ at $353 \mathrm{~K}$, and the solution part was collected by centrifugal removal of remaining solid pats.

\section{Photoirradiation and product analyses}

An aqueous suspension consisting of $15-30 \mathrm{mg}$ photocatalyst powder (15 $\mathrm{mg}$ as $\left.\mathrm{TiO}_{2}\right)$ and a $20 \mathrm{~cm}^{3}$ aqueous solutions of (a) methanol (50\%), (b) acetic acid (5\%), (c) methylene blue $\left(\mathrm{MB}, 50 \mu \mathrm{mmol} \mathrm{dm}^{-3}\right)$, or $(\mathbf{d})$ polyvinylalcohol $(\mathrm{PVA}, \mathrm{Mw}=22000,2$ $\mathrm{mg})$ was put in a Pyrex cylindrical reaction vessel $\left(6.8 \mathrm{~cm}\right.$ in diameter, $240 \mathrm{~cm}^{3}$ in capacity). In reaction a, the catalyst was platinized in situ by adding hexachloroplatinic acid $\left(\mathrm{H}_{2} \mathrm{PtCl}_{6}\right)$, the amount of which corresponded to $1 \mathrm{wt} \%$ loading of $\mathrm{Pt}$. 
Photoirradiation was performed using a $300 \mathrm{~W}$ Xe lamp from the upper part of the vessel in argon (a) or air (b, c, and d) with shaking of the suspension to achieve homogeneous dispersion. Gaseous products, $\mathrm{H}_{2}$ and $\mathrm{CO}_{2}$, were analyzed by using a Shimadzu GC-8A gas chromatograph equipped with a TCD detector and an MS-5A column (for $\mathrm{H}_{2}$ ) or a Porapak Q column (for $\mathrm{CO}_{2}$ ). It should be noted that various intermediates can be produced in these systems and it is difficult to determine the main product for PVA photodecomposition (reaction d): photocatalytic activity was evaluated by the amount of $\mathrm{CO}_{2}$ liberation for convenience. In the case of reaction c, photocatalytic activity was evaluated by decrease in MB by measuring absorbance of the solution at $650 \mathrm{~nm}\left(\varepsilon=42,000 \mathrm{dm}^{3} \mathrm{~mol}^{-1} \mathrm{~cm}^{-1}\right)$ using a Shimadzu UV-2500PC UV-vis recording spectrophotometer. For the evaluation of photocatalytic activity of alkylsilylated samples, 5-8 mg of photocatalyst powders (5 mg as $\mathrm{TiO}_{2}$ ) was added to 20 $\mathrm{cm}^{3}$ aqueous solutions of acetic acid (5\%) and phoroirradiated in air using the same equipments as that described above.

\section{Results and discussion}

\section{Structural characterization of $\mathrm{SiO}_{2} /$ void/TiO $/ \mathrm{T}_{2}$}

Figure 1 shows SEM images of bare $\mathrm{TiO}_{2}(\mathrm{ST}-41)$ and $\mathrm{TiO}_{2}$ after various modifications. The $\mathrm{TiO}_{2}$ sample without any modifications exhibited angular morphology with particle sizes of ca. 100-300 nm (Fig. 1a). Hydrothermal treatment of the sample in aqueous glucose produced a layer of ca. $60 \mathrm{~nm}$ in thickness that entirely covered the surface of $\mathrm{TiO}_{2}$ particles, as shown in Fig. 1b. Sun and Li reported that glucose was converted into carbonaceous polysaccharide (PS) spheres by hydrothermal

treatment at temperatures between $433 \mathrm{~K}$ and $453 \mathrm{~K} .{ }^{29}$ Moreover, in the presence of certain oxide particles during the hydrothermal reaction of glucose, we have demonstrated the formation of a uniform layer of PS on these particles without forming 
such PS spheres. ${ }^{30,31}$ Hence, the surface layer on $\mathrm{TiO}_{2}$ is ascribed to the formation a PS layer. The PS layer could be converted to an amorphous carbon layer by heat treatment at $873 \mathrm{~K}$ under evacuation to form $\mathrm{C} / \mathrm{TiO}_{2} .{ }^{32}$

As shown in Fig. 1c, successive treatments of $\mathrm{C} / \mathrm{TiO}_{2}$ with AEAPS and TEOS in ethanol led to coverage of an outer layer on the $\mathrm{C} / \mathrm{TiO}_{2}$ sample. From the fact that almost no free silica nanoparticles were observed, this indicates surface coverage with a silica layer by exclusive progress in polycondensation reaction at the surface of $\mathrm{C} / \mathrm{TiO}_{2}$, resulting in the formation of the $\mathrm{SiO}_{2} / \mathrm{C} / \mathrm{TiO}_{2}$ composite. On the other hand, uniform coverage of a silica layer was not achieved when $\mathrm{C} / \mathrm{TiO}_{2}$ particles without agitation in the AEAPS solution was employed; instead, irregularly shaped silica aggregates were formed on the $\mathrm{C} / \mathrm{TiO}_{2}$ surface and in the surrounding medium (Fig. 1d). These results imply that selective and complete silica coverage is promoted by the preloaded AEAPS molecules on the surface of $\mathrm{C} / \mathrm{TiO}_{2}$. Although the details of adsorption state(s) and molecular scale function(s) of AEAPS are not known, the surface AEAPS molecules probably induce a strong attractive force between the $\mathrm{C} / \mathrm{TiO}_{2}$ surface and silica to form uniform coverage of a silica layer.

In order to remove carbon components from $\mathrm{SiO}_{2} / \mathrm{C} / \mathrm{TiO}_{2}$, the sample was heated at $873 \mathrm{~K}$ in air. From the fact that no apparent weight loss accompanied by an exothermic event was observed on TG-DTA of thus-obtained white powder, complete removal of carbon components should be achieved during the heat treatment of the sample. The SEM image of the sample shown in Fig. 2a indicates no significant change in the surface morphology compared to that of $\mathrm{SiO}_{2} / \mathrm{C} / \mathrm{TiO}_{2}$ (see Fig. 1c), while the outer layer becomes semi-transparent. As expected from the SEM observation, the corresponding TEM image of the sample shows successful formation of the desired $\mathrm{SiO}_{2} /$ void $/ \mathrm{TiO}_{2}$ particles, i.e., a lateral shell of ca. $80 \mathrm{~nm}$ in thickness with encapsulated $\mathrm{TiO}_{2}$ particles and the presence of a void space with a width of a few tens nm between the $\mathrm{TiO}_{2}$ core and the $\mathrm{SiO}_{2}$ shell (Fig. 2b). When the other commercial $\mathrm{TiO}_{2}$, Degussa 
P-25, was employed, a similar $\mathrm{SiO}_{2} /$ void $/ \mathrm{TiO}_{2}$ composite was also obtained by the same procedure as that described above (Fig. 2c), implying applicability of the present method for fabrication of the $\mathrm{SiO}_{2} /$ void/TiO 2 composite to various $\mathrm{TiO}_{2}$ samples.

Figure 3 shows XRD patterns of $\mathrm{SiO}_{2} /$ void/ $\mathrm{TiO}_{2}$ samples derived from Ishihara ST-41 and Degussa P-25. XRD patterns of bare $\mathrm{TiO}_{2}$ samples are also shown for comparison. The XRD pattern of ST-41 was assigned to pure anatase and no significant change in its pattern was observed after the formation of $\mathrm{SiO}_{2} /$ void $/ \mathrm{TiO}_{2}$ : it shows almost the same widths and relative intensities of all peaks as those of bare ST-41. In the case of P-25, it is difficult to determine the existence of structural alteration because of the presence of both anatase and rutile phases in the bare sample. However, when fractions of anatase $\left(f_{A}\right)$ and rutile from integrated areas of the anatase (101) and rutile (110) peaks $^{33}$ in the XRD pattern were introduced as a convenient evaluation of the structural change, the $\mathrm{f}_{\mathrm{A}}$ value did not change after the formation of $\mathrm{SiO}_{2} /$ void $/ \mathrm{TiO}_{2}$ $\left(\mathrm{f}_{\mathrm{A}}=82 \%\right)$, indicating almost no appreciable structural difference between bare P-25 and $\mathrm{SiO}_{2} /$ void $/ \mathrm{TiO}_{2}$.

Figure 4 shows a $\mathrm{N}_{2}$ adsorption-desorption isotherm of $\mathrm{SiO}_{2} /$ void/ $\mathrm{TiO}_{2}$ derived from ST-41 and that of bare ST-41. In comparison with the isotherm of the bare sample, one of the most notable features observed in the isotherm of $\mathrm{SiO}_{2} /$ void $/ \mathrm{TiO}_{2}$ is the presence of a substantial hysteresis loop that is closed by a drop of the desorption branch in the volume adsorbed at $P / P_{0}$ of ca. 0.5 . A similar isotherm was also obtained by measurement of the $\mathrm{SiO}_{2} /$ void/ $\mathrm{TiO}_{2}$ sample fabricated from Degussa P-25. Previous studies have proved that this phenomenon is often referred to as the tensile strength effect (TSE) and is typically observed in samples having a mesopore or a macropore encapsulated by micropore systems. ${ }^{34}$ These results, therefore, suggest that the lateral silica shell not only forms a hollow structure but also has microporous systems. Indeed, application of the Saito-Foley (SF) model, which is often used for silica-based microporous materials such as zeolites and silica-gels to determine micropore-size 
distribution, to the adsorption branch results in specifying the pore system mainly ranged between ca. $0.7 \mathrm{~nm}$ and ca. $1.5 \mathrm{~nm}$ (inset of Fig. 4). For fabrication of hollow silica spheres based on the similar method reported in the literature, i.e., base-catalyzed hydrolysis and polycodensatoion of TEOS, silica shells were composed of aggregates of silica nanoparticles. ${ }^{35,36}$ Therefore, the observed microporous structure on $\mathrm{SiO}_{2} /$ void $/ \mathrm{TiO}_{2}$ is attributable to the vacant spaces between these aggregates. The successful etching of the medial carbon shell in $\mathrm{SiO}_{2} / \mathrm{C} / \mathrm{TiO}_{2}$ as described above is also attributable to such a micorporous structure of the lateral silica shell.

Specific surface areas of $\mathrm{SiO}_{2} /$ void $/ \mathrm{TiO}_{2}$ samples derived from ST-41 and P-25 determined by application of the Brunauer-Emmett-Teller (BET) method to the isotherm were $18 \mathrm{~m}^{2} \mathrm{~g}^{-1}$ and $30 \mathrm{~m}^{2} \mathrm{~g}^{-1}$, respectively. From the surface areas of bare $\mathrm{TiO}_{2}$ samples $\left(13 \mathrm{~m}^{2} \mathrm{~g}^{-1}\right.$ (ST-41) and $\left.45 \mathrm{~m}^{2} \mathrm{~g}^{-1}(\mathrm{P}-25)\right)$ and the contents of $\mathrm{SiO}_{2}$ in $\mathrm{SiO}_{2} /$ void $/ \mathrm{TiO}_{2}$, determined using ICP-AES of silicon (ca. 50\% (ST-41) and 40\% (P-25)), specific surface areas of lateral silica shells were estimated to be $23 \mathrm{~m}^{2} \mathrm{~g}^{-1}$ (ST-41) and $20 \mathrm{~m}^{2} \mathrm{~g}^{-1}$ (P-25), respectively. The similarity of these values indicates structural uniformity of the silica shell formed by the present method regardless of the kind of $\mathrm{TiO}_{2}$ core.

\section{Photocatalytic reactions on $\mathrm{SiO}_{2} /$ void/ $/ \mathrm{TiO}_{2}$}

Figure 5 shows time course curves of $\mathrm{H}_{2}$ liberation on $\mathrm{TiO}_{2}(\mathrm{ST}-41)$ and those after various modifications from an aqueous methanol solution. It is known that the $\mathrm{TiO}_{2}$ photocatalyst utilized for the reaction is generally formed by the combination with a reduction catalyst such as Pt fine particles owing to the low catalytic ability of the surface of $\mathrm{TiO}_{2}$ for $\mathrm{H}_{2}$ production. In fact, a linear increase in the amount of $\mathrm{H}_{2}$ was observed on $\mathrm{TiO}_{2}$ suspended in an aqueous methanol solution containing $\mathrm{H}_{2} \mathrm{PtCl}_{6}$, a source of Pt fine particles, while there was almost no $\mathrm{H}_{2}$ liberation in the absence of $\mathrm{H}_{2} \mathrm{PtCl}_{6}$ (Fig. 5a). ${ }^{37}$ Moreover, the direct surface coverage with a silica layer strongly 
suppressed $\mathrm{H}_{2}$ liberation: the $\mathrm{SiO}_{2} / \mathrm{TiO}_{2}$ sample showed lower activity than that of bare $\mathrm{TiO}_{2}$ (Fig. 5b). It is likely that such silica coverage prevented the adsorption of substances participating in the reaction, such as methanol, $\mathrm{H}_{2} \mathrm{O}$ and $\mathrm{H}_{2} \mathrm{PtCl}_{6}$. On the other hand, despite the presence of an appreciable amount of silica components (see above), the $\mathrm{SiO}_{2} /$ void $/ \mathrm{TiO}_{2}$ sample showed almost the same activity as that of the bare $\mathrm{TiO}_{2}$ sample, as shown in Fig. 5b. TEM observation of the sample after the reaction shown in Fig. 6 clearly indicates deposition of $\mathrm{Pt}$ fine particles on the medial $\mathrm{TiO}_{2}$ without any collapse of the silica shell. Thus, the unusual photocatalytic activity observed on $\mathrm{SiO}_{2} /$ void/TiO ${ }_{2}$ is attributable to the presence of the pore system and the void space in the silica shell and between the shell and medial $\mathrm{TiO}_{2}$ particles, respectively. i.e., these structures led to efficient mass transfers through the silica shell to supply substances that participate in this reaction to the naked active surface of the medial $\mathrm{TiO}_{2}$ core when the sizes of these substances were smaller than the pore system of the silica shell shown in the inset of Fig. 4.

In order to investigate in detail the function of the silica shell in $\mathrm{SiO}_{2} /$ void/ $/ \mathrm{TiO}_{2}$, photocatalytic activity was evaluated by degradation of various substrates with different sizes in aqueous suspension systems. Typical results together with those for bare $\mathrm{TiO}_{2}$ and $\mathrm{SiO}_{2} / \mathrm{TiO}_{2}$ are summarized in Table 1. For the decomposition of acetic acid, $\mathrm{SiO}_{2} /$ void $/ \mathrm{TiO}_{2}$ showed activity almost the same as that of bare $\mathrm{TiO}_{2}$, similar to the above-described results for $\mathrm{H}_{2}$ liberation. On the other hand, in the degradation of $\mathrm{MB}$, the molecular size of which is close to the pore system of the silica shell, $\mathrm{SiO}_{2} /$ void $/ \mathrm{TiO}_{2}$ showed lower activity than that of bare $\mathrm{TiO}_{2}$. Moreover, for the degradation of a large substrate, i.e., PVA, only a small amount of $\mathrm{CO}_{2}$ was liberated on $\mathrm{SiO}_{2} /$ void $/ \mathrm{TiO}_{2}$ despite liberation of a large amount of $\mathrm{CO}_{2}$ on bare $\mathrm{TiO}_{2}$. The suppression of photocatalytic activity for degradation of such relatively large molecules compared to the activity of bare $\mathrm{TiO}_{2}$ clearly indicates a blocking effect of the lateral silica shell to prevent diffusion of such large substrates moving into the shell to reach 
the surface of the medial $\mathrm{TiO}_{2}$ particles. These results indicate that use of the $\mathrm{SiO}_{2} /$ void $/ \mathrm{TiO}_{2}$ structure is a promising strategy for designing novel photocatalysts with significant molecular selective properties without any reduction in intrinsic activity of $\mathrm{TiO}_{2}$. In addition, as expected from the results for $\mathrm{H}_{2}$ liberation, the $\mathrm{SiO}_{2} / \mathrm{TiO}_{2}$ sample showed poor activities for all of the reactions tested owing to the direct surface coverage with the silica layer (entry 3).

In our previous study, we have proposed unique photocatalytic systems based on photocatalyst particles assembled at oil-water and gas-water interfaces. ${ }^{30,38}$ For these systems, we employed $\mathrm{TiO}_{2}$ particles coated with porous silica, the surface of which was modified with a hydrophobic silane agent: indirect attachment of the hydrophobic agent to the surface of $\mathrm{TiO}_{2}$ enables inhibition of photocatalytic decomposition of such an agent. The properties of the $\mathrm{SiO}_{2} /$ void $/ \mathrm{TiO}_{2}$ photocatalysts described above indicate the possibility of utilizing those photocatalysts for such photocatalytic systems.

Based on these facts and considerations, we investigated the properties and photocatalytic activity of $\mathrm{SiO}_{2} /$ void $/ \mathrm{TiO}_{2}$ photocatalysts (derived from Degussa P-25) loaded with alkylsilyl groups $\left(\mathrm{o}-\mathrm{SiO}_{2} /\right.$ void $\left./ \mathrm{TiO}_{2}\right)$. Fig. 7a shows photographs of o- $\mathrm{SiO}_{2} /$ void $/ \mathrm{TiO}_{2}$ added to pure water before and after photoirradiation for certain periods. While the $\mathrm{SiO}_{2} /$ void $/ \mathrm{TiO}_{2}$ particles without loading of alkylsilyl groups were dispersed in water due to their hydrophilic surface nature (data not shown), o- $\mathrm{SiO}_{2} /$ void $/ \mathrm{TiO}_{2}$ floated on the gas-water interface: the particles became hydrophobic due to the surface coverage of alkylsilyl groups. When the bare $\mathrm{TiO}_{2}(\mathrm{P}-25)$ sample was treated with ODS for comparison $\left(\mathrm{o}-\mathrm{TiO}_{2}\right)$, a similar hydrophobic property also appeared, i.e., visual observation showed practically no difference between o- $\mathrm{SiO}_{2} /$ void $/ \mathrm{TiO}_{2}$ and $\mathrm{o}-\mathrm{TiO}_{2}$, as shown in Fig. $7 \mathrm{~b}$. On the other hand, a significant difference was observed between them upon photoirradiation of these samples: most of o- $\mathrm{SiO}_{2} /$ void $/ \mathrm{TiO}_{2}$ particles floated at the interface, while o- $\mathrm{TiO}_{2}$ gradually settled during the photoirradiation. Previous studies have shown the inability of o- $\mathrm{TiO}_{2}$ to float on the 
gas-water interface is due attributed to photodecomposition of alkylsilyl groups on the surface through the photocatalytic reaction of $\mathrm{TiO}_{2}{ }^{39}$

Further photoirradiation of the o-SiO $2 /$ void $/ \mathrm{TiO}_{2}$ sample induced gradual sinking of the sample into the aqueous phase. However, the fact that most of the recovered sample again floated at the gas-water interface ${ }^{40}$ implies retention of most of the loaded alkylsilyl groups on the o- $\mathrm{SiO}_{2} /$ void $/ \mathrm{TiO}_{2}$ sample. Although we have no clear evidence at present, a possible explanation for such photosinking of o- $\mathrm{SiO}_{2} /$ void $/ \mathrm{TiO}_{2}$ is that an increase in $\mathrm{TiOH}$ during the photoirradiation, i.e., the formation of a superhydrophilic surface of the medial $\mathrm{TiO}_{2}$ induced by band-gap excitation of $\mathrm{TiO}_{2},{ }^{10,11}$ leads to alteration of the hydrophilic-hydrophobic balance of $\mathrm{o}-\mathrm{SiO}_{2} /$ void/ $\mathrm{TiO}_{2}$ toward the hydrophilic side.

Floating photocatalysts are useful for water purification because of the ease of separation from the solution and efficient utilization of irradiated photon(s) even though presences of components interrupting the light in the solution. ${ }^{41-44}$ Thus, we studied the photocatalytic function of o- $\mathrm{SiO}_{2} /$ void $/ \mathrm{TiO}_{2}$ for decomposition of aqueous acetic acid as a model reaction. Figure 8 shows time course curves of $\mathrm{CO}_{2}$ liberation on o- $-\mathrm{SiO}_{2} /$ void $/ \mathrm{TiO}_{2}$. The results for o- $\mathrm{TiO}_{2}$ under the same conditions and results for $\mathrm{TiO}_{2}$ in the suspension system are also shown in this figure. Despite the fact that most of the particles floated at the gas-water interface during the irradiation period (see Fig. 7), o- $\mathrm{TiO}_{2}$ showed lower activity than the activities of other samples. As described for the $\mathrm{SiO}_{2} / \mathrm{TiO}_{2}$ sample employed in above suspension systems, the suppression of photocatalytic activity is ascribed to the coverage of surface active site(s) with alkylsilane components. On the other hand, the o- $\mathrm{SiO}_{2} /$ void $/ \mathrm{TiO}_{2}$ sample that floated at the gas-water interface showed ca. $80 \%$ of the activity of the bare $\mathrm{TiO}_{2}$ suspension, as was expected from its specific structure described above. The slight decrease in activity is probably due to the partial coverage with alkylsilyl groups on the medial $\mathrm{TiO}_{2}$ and/or the shielding of a part of pore systems in the lateral silica shell. 


\section{Conclusion}

In this study, we have shown that $\mathrm{SiO}_{2} /$ void/ $/ \mathrm{TiO}_{2}$ composites, commercially available $\mathrm{TiO}_{2}$ photocatalyst particles encapsulated in hollow silica shells, have size-selective photocatalytic functions for various reactions. Such properties will bring about a revolution in the practical utility of $\mathrm{TiO}_{2}$ photocatalysis for incorporation in various organic products such as fibers, polymers and paints. The basic concept of the present core-shell photocatalyst is also a promising strategy for designing photocatalysts with significant molecular selective properties toward organic synthesis when the pore diameter and distribution of the shells are controlled. Moreover, efficient control of the hydrophobic property of the shell led to flotation of the composites, indicating potential applications for water purification. Thus, we can expect further development of a new category of photocatalytic systems based on the present $\mathrm{SiO}_{2} /$ void/ $/ \mathrm{TiO}_{2}$ composites when further controls and optimizations of the structure, such as pore diameters/distributions and hydrophobic characters of silica shells and kinds of photocatalysts used, are attained.

\section{Acknowledgements}

This work was supported by a Grant-in-Aid for Scientific Research on Priority Areas (No. 19028042, "Chemistry of Concerto Catalysis") from the Ministry of Education, Culture, Sports, Science and Technology, Japan. Dr. Takao Sakata and Professor Hirotaro Mori (Osaka University) are gratefully acknowledged for their help in TEM measurements. The authors would also like to thank Professor Takayuki Hirai (Osaka University) for permission to use an ICP-AES. 


\section{References}

1. P. V. Kamat, Chem. Rev., 2003, 93, 267.

2. T. Hirakawa, H. Kominami, B. Ohtani and Y. Nosaka, J. Phys. Chem. B, 2001, 105, 6993.

3. P. Pichat, C. Guillard, C. Maillard, L. Amalric and J. C. D'Oliveira, Trace Met. Environ.,1993, 3, 207.

4. R. I. Bickley, R. K. M. Jayanty, V. Vishwanathan and J. A. Navio, NATO ASI Ser., Ser. C, 1986, 174, 555 .

5. M. R. Hoffmann, S. T. Martin, W. Choi and D. W. Bahnemann, Chem. Rev.,1995, 95, 69.

6. A. Fujishima, K. Hashimoto and T. Watanabe, $\mathrm{TiO}_{2}$ Photocatalysis-Fundamentals and Applications, BKC, Tokyo, 176 (1999).

7. A. Fujishima, T. N. Rao and D. A. Tryk, J. Photochem. Photobiol. C: Photochem. Rev., 2000, 1, 1.

8. R. Asahi, T. Morikawa, T. Okwaki, K. Aoki and Y. Taga, Science, 2001, 293, 269.

9. R. Wang, K. Hashimoto, A. Fujishima, M. Chikuni, E. Kojima, A. Kitamura, M. Shimohigoshi and T. Watanabe, Nature, 1997, 388, 431.

10. A. Nakajima, K. Hashimoto, T. Watanabe, K. Takai, G. Yamauchi and A. Fujishima, Langmuir, 2000, 16, 7044.

11. N. Sakai, A. Fujishima, T. Watanabe and K. Hashimoto, J. Phys. Chem. B, 2003, 107, 1028.

12. M. A. Fox and M. J. Chen, J. Am. Chem. Soc., 1983, 105, 4497.

13. T. Ohno, K. Nakabeya and M. Matsumura, J. Catal., 1998, 76, 176.

14. T. Ohno, T. Kigoshi, K. Nakabeya and M. Matsumura, Chem. Lett., 1998, 877.

15. S. Higashida, A. Harada, R. Kawakatsu, N. Fujiwara and M. Matsumura, Chem. Commun., 2006, 2804.

16. J. G. Yu, J. C. Yu, M. K. P. Leung, W. K. Ho, B. Cheng, X. J. Zhao and J. C. Zhao, J. 
Catal., 2003, 217, 69.

17. Y. Shiraishi, N. Saito and T. Hirai, J. Am. Chem. Soc., 2005, 127, 12820.

18. Y. M. Xu and C. H. Langford, J. Phys. Chem. B, 1997, 101, 3115.

19. L. Davydov, E. P. Reddy, P. France and P. G. Smirniotis, J. Catal., 2001, 203, 157.

20. G. Cosa, M. S. Galletero, L. Fernández, F. Márquez, H. García and J. C. Scaiano, New J. Chem., 2002, 26, 1448

21. F. X. L. Xamena, P. Calza, C. Lamberti, C. Prestipino, A. Damin, S. Bordiga, E. Pelizzetti and A. Zecchina, J Am. Chem. Soc., 2003, 125, 2264.

22. M. Morishita, Y. Shiraishi and T. Hirai, J. Phys. Chem. B, 2006, 110, 17898.

23. S. Ikeda, Y. Kowata, K. Ikeue, M. Matsumura and B. Ohtani, Appl. Catal. A, 2004, 265, 69.

24. K. Inumaru, T. Kasahara, M. Yasui and S. Yamanaka, Chem. Commun., 2005, 2131.

25. S. Shanmugam, A. Gabashvili, D. S. Jacob, J. C. Yu and A. Gedanken, Chem. Mater, 2006, 18, 2275.

26. T. Ohno, T. Tsubota, K. Kakiuchi, S. Miyayarna and K. Sayama, J. Mol. Catal. A., 2006, 245, 47.

27. S. Ikeda, Y. Ikoma, H. Kobayashi, T. Harada, B. Ohtani, T. Torimoto and M. Matsumura, Chem Commun., DOI:10.1039/B704468B.

28. C. Graf, D. L. J. Vossen, A. Imhof and A. van Blaaderen, Langmuir, 2003, 19, 6693.

29. X. Sun and Y. Li, Angew. Chem. Int. Ed., 2004, 43, 597; Langmuir, 2005, 21, 6019.

30. S. Ikeda, K. Hirao, S. Ishino, M. Matsumura and B. Ohtani, Catal. Today, 2006, 117, 343.

31. S. Ikeda, K. Tachi, Y. H. Ng, Y. Ikoma, T. Sakata, H. Mori, T. Harada, M. Matsumura, Chem. Mater, in press.

32. Note that no distinct alteration of surface morphology was induced by this treatment (data not shown).

33. T. Torimoto, N. Nakamura, S. Ikeda and B. Ohtani, Phys. Chem. Chem. Phys., 2002, 
4, 5910.

34. J. C. Groen, L. A. A. Peffer and J. Pérez-Ramírez, Microporous Mesoporous Mater, 2003, 60, 1 .

35. I. Tissot, J. P. Reymond, F. Lefebvre and E. Bourgeat-Lami, Chem. Mater, 2002, 14, 1325

36. Y. Lu, J. McLellan and Y. Xia, Langmuir, 2004, 20, 3464.

37. In this system, photoexcited electrons $\left(\mathrm{e}^{-}\right)$reduce $\mathrm{PtCl}_{6}{ }^{2-}$ ions to make $\mathrm{Pt}$ deposits which act as reduction catalysis of water using e $\mathrm{e}^{-}$to produce $\mathrm{H}_{2}$.

38. S. Ikeda, Y. Kowata, K. Ikeue, M. Matsumura and B. Ohtani, Appl. Catal. A, 2004, 265, 69.

39. T. Kato, A. Fujishima, E. Maekawa and K. Honda, Nihon Kagaku Kaishi, 1986, 1, 8. 40. After photoirradiation for several hours, the o- $\mathrm{SiO}_{2} /$ void/ $/ \mathrm{TiO}_{2}$ sample was collected and dried at $353 \mathrm{~K}$ in air overnight. Then the dried sample was again added to the pure water. Note that most of $\mathrm{o}-\mathrm{TiO}_{2}$ recovered by the same procedure did not achieve such re-floatation.

41. H. Al-Ekabi and N. Serpone, J. Phys. Chem., 1988, 92, 5726.

42. G. Dagan and M. Tomkiewicz, J. Phys. Chem., 1993, 97, 12651.

43. G. Dagan, S. Sampath and O. Lev, Chem. Mater., 1995, 7, 446.

44. K. Ramanathan, D. Avnir, A. Modestov and O. Lev, Chem. Mater., 1997, 9, 2533.

\section{Figure Captions}

Figure 1. SEM images of (a) $\mathrm{TiO}_{2}(\mathrm{ST}-41)$ and (b) that after hydrothermal treatment with an aqueous glucose solution. (c) SEM image of $\mathrm{C} / \mathrm{TiO}_{2}$ after successive treatment with AEAPS and TEOS. (d) SEM image of $\mathrm{C} / \mathrm{TiO}_{2}$ after treatment with TEOS. Scale bars correspond to $200 \mathrm{~nm}$.

Figure 2. (a) SEM image and (b) TEM image of powders after heat treatment of 
$\mathrm{SiO}_{2} / \mathrm{C} / \mathrm{TiO}_{2}$ derived from $\mathrm{ST}-41$ at $873 \mathrm{~K}$ in air. (c) TEM image of the sample derived from P-25. Scale bars correspond to $200 \mathrm{~nm}$.

Figure 3. XRD patterns of (a) $\mathrm{TiO}_{2}(\mathrm{ST}-41)$, (b) $\mathrm{SiO}_{2} /$ void $/ \mathrm{TiO}_{2}$ derived from ST-41, and (c) Degussa P-25 and $\mathrm{SiO}_{2} /$ void $/ \mathrm{TiO}_{2}$ derived from P-25.

Figure 4. $\mathrm{N}_{2}$ adsorption-desorption isotherms of (a) $\mathrm{TiO}_{2}(\mathrm{ST}-41)$ and $\mathrm{SiO}_{2} /$ void $/ \mathrm{TiO}_{2}$ derived from ST-41. Filled and open circles denote adsorption and desorption branches, respectively. The inset shows corresponding micropore-size distributions calculated by using the SF method.

Figure 5. (a) Time course curves of $\mathrm{H}_{2}$ liberation on $\mathrm{TiO}_{2}(\mathrm{ST}-41)$ from a deaerated aqueous methanol solution in the presence (open circles) or absence (filled circles) of $\mathrm{H}_{2} \mathrm{PtCl}_{6}$. (b) Time course curves of $\mathrm{H}_{2}$ liberation on $\mathrm{SiO}_{2} / \mathrm{TiO}_{2}$ (open triangles) and $\mathrm{SiO}_{2} /$ void $/ \mathrm{TiO}_{2}$ (filled triangles) derived from ST-41 from a deaerated aqueous methanol solution containing $\mathrm{H}_{2} \mathrm{PtCl}_{6}$.

Figure 6. TEM image of the $\mathrm{SiO}_{2} /$ void $/ \mathrm{TiO}_{2}$ (ST-41) sample after photoirradation in aqueous methanol containing $\mathrm{H}_{2} \mathrm{PtCl}_{6}$. Scale bars correspond to $200 \mathrm{~nm}$.

Figure 7. Locations of (a) $0-\mathrm{SiO}_{2} /$ void/ $/ \mathrm{TiO}_{2}(\mathrm{P}-25)$ and (b) $\mathrm{o}-\mathrm{TiO}_{2}(\mathrm{P}-25)$ added to water before $(0 \mathrm{~h})$ and after photoirradiation for some periods ( $1 \mathrm{~h}$ and $3 \mathrm{~h}$ ).

Figure 8. Time course curves of $\mathrm{CO}_{2}$ liberation on $\mathrm{TiO}_{2}$ (P-25) (open circles), o- $\mathrm{TiO}_{2}$ (filled circles) and $\mathrm{o}-\mathrm{SiO}_{2} /$ void $/ \mathrm{TiO}_{2}$ (open triangles) from an aerated aqueous acetic acid solution. 


\section{Graphics for the contents list}

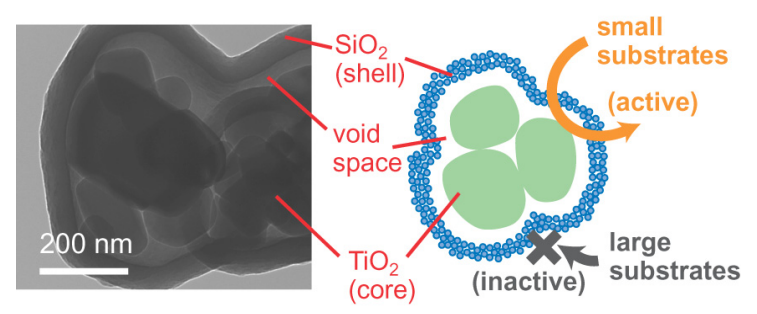

A novel core-shell composite of titanium(IV) oxide $\left(\mathrm{TiO}_{2}\right)$ particles directly incorporated into a hollow silica shell was fabricated. The composite induced efficient photocatalytic reactions when relatively small substrates were used, such as methanol dehydration and decomposition of acetic acid, without any reduction in the intrinsic activity of original $\mathrm{TiO}_{2}$ but did not exhibit efficient photocatalytic activity for decomposition of large substrates, methylene blue and polyvinylalcohol. The loading of alkylsilyl groups on the surface of the composite led to highly photostable floatability: the floated sample also induced efficient photocatalytic reaction for decomposition of acetic acid while retaining floatation at the gas-water interface.

Table 1. Photocatalytic activity of $\mathrm{TiO}_{2}(\mathrm{ST}-41)$ and that after various modifications for decomposition of various substrates ${ }^{\mathrm{a}}$

\begin{tabular}{lllll}
\hline entry & photocatalyst & $\begin{array}{l}\mathrm{R}_{\mathrm{CO} 2}(\mathrm{AcOH})^{\mathrm{b}} \\
/ \mu \mathrm{mol} \mathrm{h}^{-1}\end{array}$ & $\begin{array}{l}\mathrm{D}_{\mathrm{MB}}{ }^{\mathrm{d}} \\
/ \mu \mathrm{mol}\end{array}$ & $\begin{array}{l}\mathrm{R}_{\mathrm{CO} 2}(\mathrm{PVA})^{\mathrm{d}} \\
/ \mu \mathrm{mol} \mathrm{h}^{-1}\end{array}$ \\
\hline 1 & $\mathrm{TiO}_{2}$ & 87.4 & 0.65 & 27.2 \\
2 & $\mathrm{SiO}_{2} /$ void $/ \mathrm{TiO}_{2}$ & 84.2 & 0.34 & 5.6 \\
3 & $\mathrm{SiO}_{2} / \mathrm{TiO}_{2}$ & 2.8 & 0.07 & 0.2
\end{tabular}

a Photoirradiation was carried out in aqueous suspension consisting of $15-30 \mathrm{mg}$ photocatalyst powder (15 $\mathrm{mg}$ as $\left.\mathrm{TiO}_{2}\right)$ and a $20 \mathrm{~cm}^{3}$ aqueous solutions of acetic acid (5\%), MB $\left(50 \mu \mathrm{mol} \mathrm{dm}{ }^{-3}\right)$, or PVA $(2 \mathrm{mg})$.

${ }^{\mathrm{b}}$ Rate of $\mathrm{CO}_{2}$ liberation from aqueous acetic acid solution. ${ }^{\mathrm{c}}$ Amount of MB decreased after 1-h photoirradiation. ${ }^{\mathrm{c}}$ Rate of $\mathrm{CO}_{2}$ liberation from an aqueous PVA solution. 

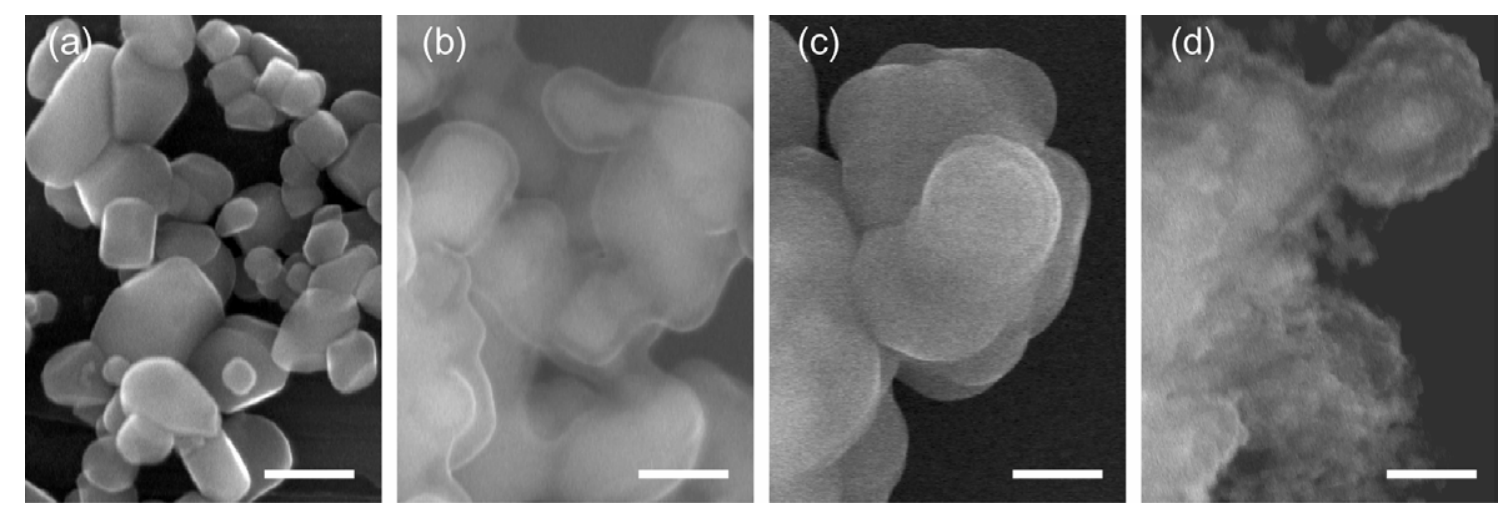

Fig. 1
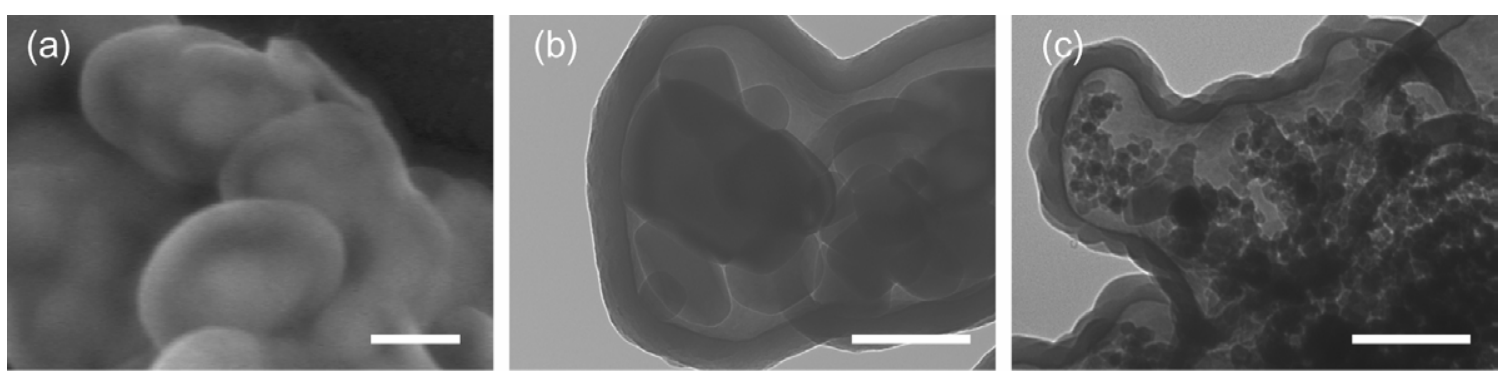

Fig. 2 


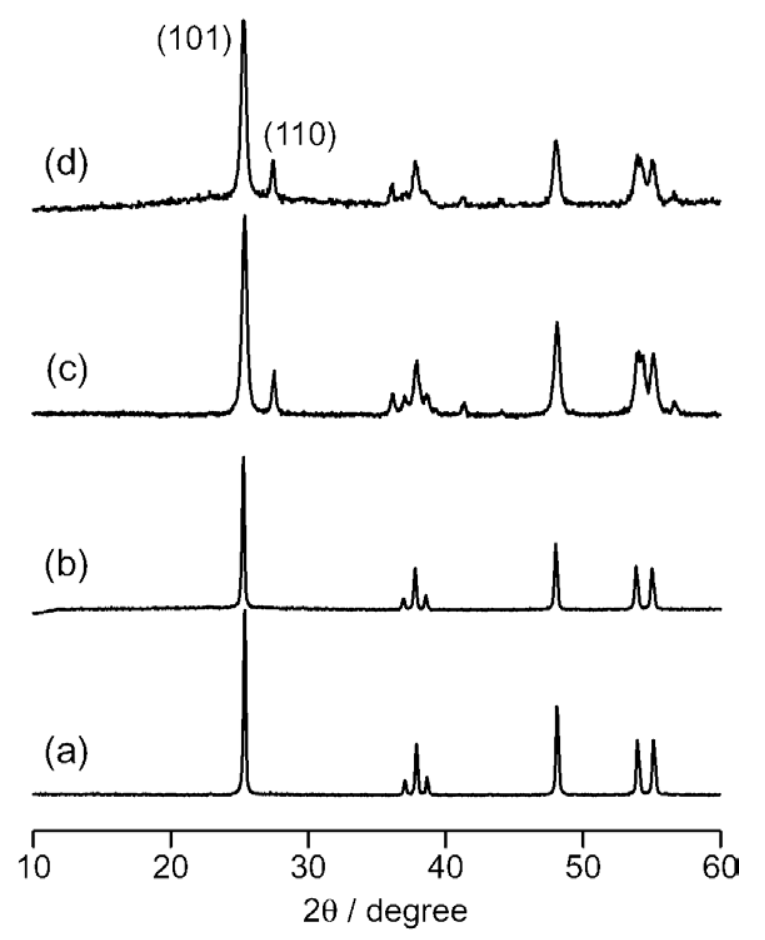

Fig. 3

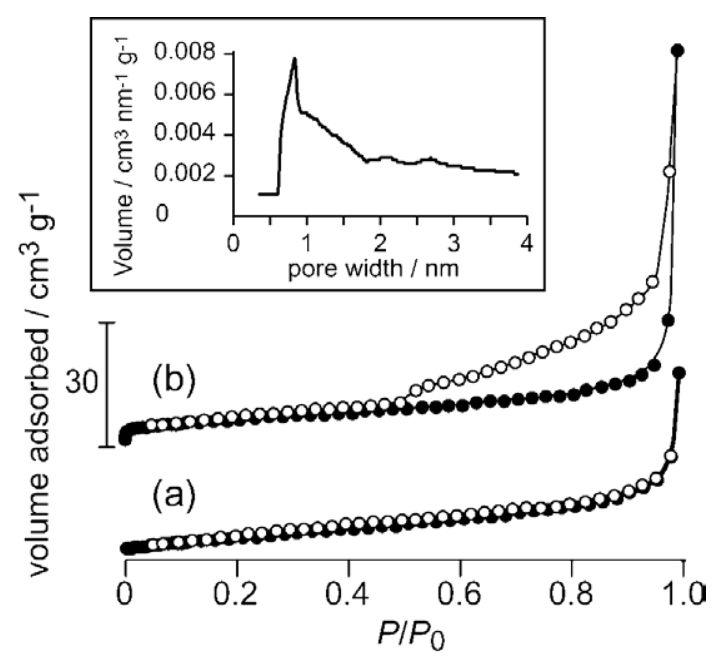

Fig. 4 


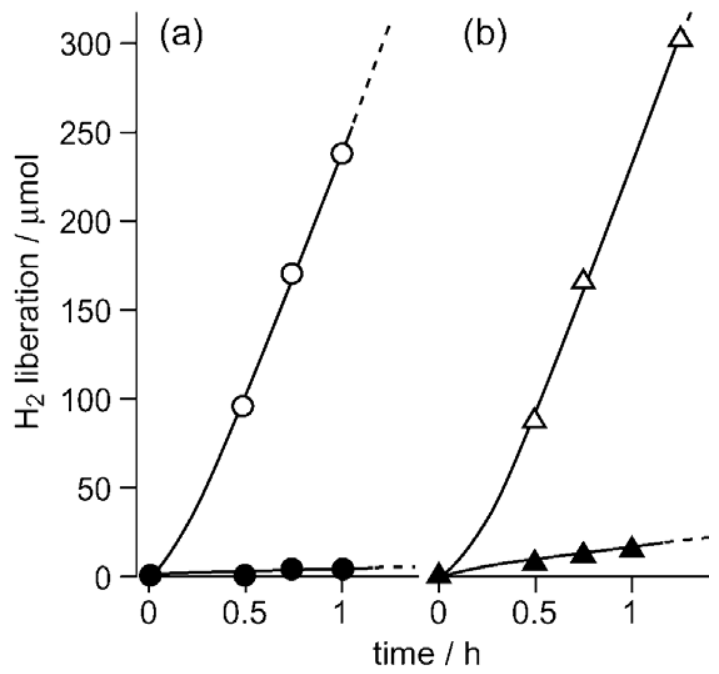

Fig. 5

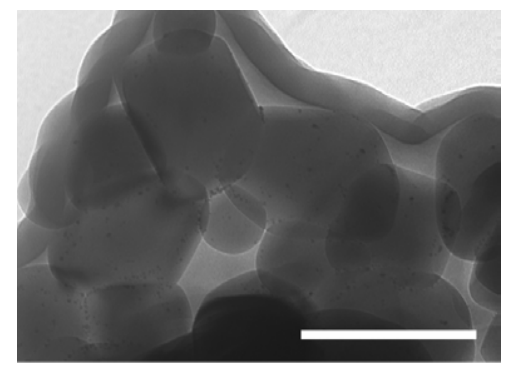

Fig. 6 


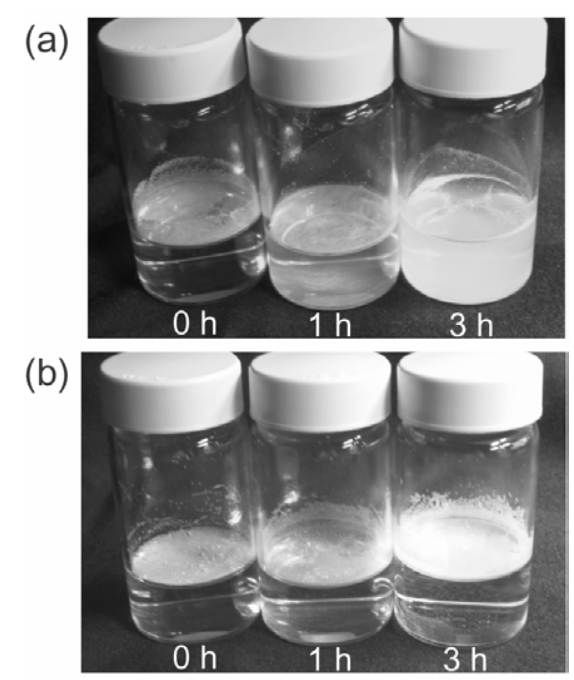

Fig. 7

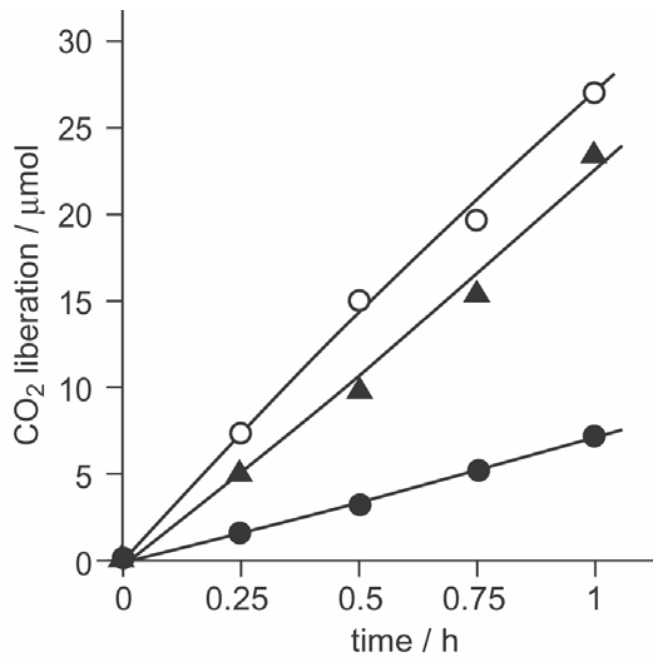

Fig. 8 